\title{
On mending the genome and mentoring the epigenome
}

\author{
David Albertini
}

Published online: 24 May 2011

(C) Springer Science+Business Media, LLC 2011

We are quickly approaching the tenth anniversary of the publication of the complete human genome in the journal, Science. This monumental and costly effort involved sequencing over 3 billion nucleotides; this resulted in the identification of a mere 20,000 protein-encoding genes (accounting for $30,000,000$ nucleotides, or 1/100th of the total human genome). Last year alone, nearly 100,000 members of our species had their genomes sequenced, owing to the extraordinary advances in sequencing technology that have emerged over the past ten years. Imagine the impact that sequencing individual genomes for an estimated price tag of $\$ 10,000$ per person will have on the future of human genetics! Even today, approximately 1,200 of our genes are now accessible for genetic testing of specific diseases, and this feat alone will alter the the future evaluation of the role of our genomes in fertility and infertility.

Having our genome sequence in hand offers a perspective on how our DNA contributes to human health and disease. Equally surprising as the paltry and finite collection of 20,000 genes identified, has been the revelation of the epigenome. Simply stated, what linear streams of nucleotide litanies unveil is but the core of how we manage decoding and implementing gene products-proteins that ultimately get the job done. Whether it be filtering from this vast DNA treasure chest those regions of the genome thatupon translation of their mRNAs-yield the players

Capsule Identifying the relationship between genetics and epigenetics is uncovering sources of disease provoking previously unappreciated and daunting with respect to how we keep our genomes and epigenomes in a healthy state.

D. Albertini $(\square)$

University of Kansas Medical Center,

Kansas City, KS 66103, USA

e-mail: dalbertini@kumc.edu responsible for DNA replication, damage detection and repair, or the symphonies of gene expression that drive key developmental events or underly the onset of disease states, our epigenomes make sense of our genomes on a daily basis.

Epigenomes, in the classical sense, embody the dynamics of chromatin that specify phenotypes, in the absence of changes in the genome. Only in the past ten years has the molecular interrogation of DNA complexed to histones revealed the secrets underlying epigenetic regulation of gene expression. Initially prompted by the discovery that direct covalent modification of cytosine residues form the basis for regulating the expression of imprinted genes, studies detailing a vast array of histone modifications have allowed for the enunciation of another code of (epi-)genetic regulation - the Histone Code!

Consider for a moment that mending our somatic genomes is dutifully attended to on a daily basis by the DNA damage and repair machinery harbored in each of our 1 billion cells. This machinery includes some 50 gene products whose job it is to sense, locate, and effect corrective measures involving base-pair mismatches and single- or double-strand DNA breaks; also incumbent on them is the daunting task of looking after the completely replicated genomes resulting from the S-phase of the cell cycle - no small task, indeed. Furthermore, how is this accomplished while ensuring that, each time a cell divides, it passes along faithfully replicated and mistake-free genomes to daughter cells? The answer for this and other challenges facing the preservation of our genomes lies somewhere in the actions of these gene products on the post-translational modifications in histones within the nucleosomes. Mentoring the nucleosomal gene garden disposed in our nuclei is well within the purview of epigenetics. 
With this as background, we examine several aspects of epigenetic regulation in this issue of JARG. These papers face, head-on, the perplexing case of genetic stability (or instability) as it pertains to the production of stem cells and their predicted utility in the treatment of human disease. Our treatment of this timely topic begins with a provocative call for re-examining the stem cell reservoirs that have received validation by clinicians and scientists in the past, relative to the buffet of stem-cell options that is garnering headline treatment on a regular basis. To wit, Zech and colleagues proffer consideration of the potential therapeutic value that may or may not be derived from different stemcell types that occupy current menu options.

This article is followed by a timely and thorough review of the induced pluripotent stem-cell field by Lai and colleagues. Their review focuses on the rapid development of emergent technologies for deriving such kinds of cells, since the original elegant studies of Yamanaka first drew attention to the prospects of reprogramming a somatic cell - one not too distant from the totipotent ancestors we affectionately regard as embryonic stem cells.

To the subject of epigenetics and genetic instability come two original research papers that delve into the matters of imprinted genes and the histone code in embryonic stem cells. Tuning in to those genetic and epigenetic traits whose fidelity must be maintained through successive passaging and expansion of stem-cell lines (if they are ever to reach the target of scaling proportions consistent with replacement/regenerative applications), these two papers offer somewhat contradictory data that may be attributable to the kinds and sources of the human embryonic stem cells analyzed. The paper by Gao and colleagues demonstrates both genetic and epigenetic alterations that attend passage of ES cell lines originally derived from parthenogenic embryos. It also uncovers some unusual behavior for the $\mathrm{X}$ chromosome that would be worrisome to anyone planning to use these cells for therapeutic indications. In contrast, the paper by Zhou et al documents epigenetic changes in imprinted and non-imprinted genes in ES cell lines that were derived from fertilized blastocysts. The reader is left to ponder what it may mean, should the rules of genetic or epigenetic instability play out differently in different types of stem cells, just as it appears to be in the case of human oocytes and embryos.

This a formidable obstacle inherent to the stem-cell field. In its ugliest form, sloppy quality control during the cell cycle can result in aneuploid or DNA-defective daughter cells when the mitotic or DNA-repair machinery fails in its appointed task. Whether engaged in the job of mending the genome or mentoring the epigenome, there is little room for error. This underscores a dire call for more research into this rate-limiting dimension of cellular behavior. 